\title{
INSS Stage 2B
}

National Cancer Institute

\section{Source}

National Cancer Institute. INSS Stage 2B. NCI Thesaurus. Code C85419.

The tumor is unilateral and the resection is complete or incomplete; ipsilateral lymph nodes are involved by tumor. 